\title{
Small Esophageal Varices in Patients with Cirrhosis-Should We Treat Them?
}

\author{
Thomas Reiberger $^{1,2} \cdot$ Theresa Bucsics $^{1,2} \cdot$ Rafael Paternostro $^{1,2} \cdot$ Nikolaus Pfisterer $^{1,2,3} \cdot$ Florian Riedl $^{1,2,4}$. \\ Mattias Mandorfer ${ }^{1,2}$
}

Published online: 7 November 2018

(C) The Author(s) 2018

\begin{abstract}
Purpose of Review The natural history and classification systems of small varices ( $\leq 5 \mathrm{~mm}$ in diameter) in cirrhotic patients with portal hypertension are summarized. Studies that assessed the course of and therapeutic intervention for small varices are discussed.

Recent Findings Current non-invasive methods show suboptimal sensitivity to detect small varices in patients with cirrhosis. Next to etiological therapy, hepatic venous pressure gradient (HVPG)-guided non-selective betablocker or carvedilol treatment has shown to impact on natural history of small varices.

Summary The main therapeutic focus in cirrhotic patients with small varices is the cure of the underlying etiology. The optimal management of small varices should include measurement of HVPG. A pharmacological decrease in HVPG by non-selective betablocker therapy of $\geq 10 \%$ reduces the risk of progression to large varices, first variceal bleeding, and hepatic decompensation. If HVPG is not available, we would recommend carvedilol $12.5 \mathrm{mg}$ q.d. for treatment of small varices in compensated patients without severe ascites. Only if small esophageal varices (EV) are not treated or in hemodynamic non-responders, follow-up endoscopies should be performed in 1-2 years of intervals considering the activity of liver disease or if hepatic decompensation occurs.
\end{abstract}

Keywords Small varices $\cdot$ Portal hypertension $\cdot$ Cirrhosis $\cdot$ Variceal bleeding $\cdot$ Low-risk varices

\section{Introduction}

Patients with cirrhosis (i.e., advanced chronic liver disease, ACLD) might develop clinically significant portal hypertension $(\mathrm{CSPH})$ with hepatic venous pressure gradient (HVPG) $\geq 10 \mathrm{mmHg}$. With increasing severity of portal hypertension,

This article is part of the Topical Collection on Portal Hypertension

Thomas Reiberger

thomas.reiberger@meduniwien.ac.at

1 Vienna Hepatic Hemodynamic Lab, Medical University of Vienna, Vienna, Austria

2 Division of Gastroenterology \& Hepatology, Department of Medicine III, Medical University of Vienna, Vienna, Austria

3 Division of Gastroenterology, Krankenanstalt Rudolfstiftung, Vienna, Austria

4 Division of Gastroenterology, Medicine II, Universitätsklinikum St. Pölten, St. Pölten, Austria patients are more likely to suffer from first variceal bleeding and recurrent variceal bleeding from esophageal varices $(\mathrm{EV})$. The beneficial role of non-selective betablockers (NSBB) in combination with endoscopic band ligation (EBL) in preventing recurrent variceal bleeding (secondary prophylaxis) has been clearly demonstrated in several trials [1] and meta-analyses [2]. In the setting of primary prophylaxis, there is strong evidence for a beneficial role of NSBB if EV are

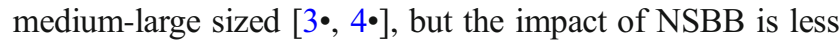
clear in patients with small EV. While there is no conclusive evidence that NSBB treatment reduces the risk of variceal bleeding or mortality in patients with small low-risk varices who have not bled [5], the absence of evidence should not be mistaken for evidence of absence. Most trials in patients with no/small varices were not sufficiently powered to detect favorable effects of NSBB on hard endpoints such as variceal bleeding or hepatic decompensation, which occur less commonly in this group of patients [6]. This is the reason why well-designed studies on clinically meaningful surrogate endpoints (e.g., variceal growth) are particularly relevant in this 
setting. Previously, there were two important trials with somewhat contradictory results: While a randomized controlled trial (RCT) by Merkel et al. [7] demonstrated that conventional NSBB (nadolol) therapy is effective in preventing the progression from small to large varices in patients who have not bled, another RCT by Sarin and co-workers [8] using propranolol reported no effect. In this review on small EV, we aim to summarize important non-invasive and invasive diagnostic aspects, pathophysiological considerations, and available evidence for pharmacological therapy to guide treatment decisions in daily clinical practice.

\section{Classification of EV: Size, Location, Presence of Red Spots, Low Versus High Risk}

In $\mathrm{CSPH}$, sub-epithelial longitudinal veins might enlarge to form varices in the esophagus. With increasing size and with rising intravascular pressure, they are more likely to rupture. Screening endoscopy is traditionally recommended to be performed in all patients after the diagnosis of cirrhosis. To timely initiate prophylaxis for variceal bleeding, early detection and specific classification of EV are major goals in the management of patients with ACLD [9]. Reports of gastroduodenoscopy performed in patients with cirrhosis must include information about the absence/presence of varices, their size, their location, and presence of red spot signs. The latter indicates sites where the variceal wall is thinning, and thus represents a sign for increased risk of EV rupture and bleeding.

Historically, the first endoscopic classification was developed by Brick and Palmer in 1964 [10]. Over time, other classifications were presented, with several ways to describe the size/grade of varices [11-13]. Based on these classifications, varices were described as grades I to IV, or more intuitively, as small, medium, or large. The presence of risk factors for variceal rupture was described as red wale signs, cherry spots, red spots, or by similar terminology. The recently updated Austrian Billroth III consensus simplified the grading of EV size just into small $(<5 \mathrm{~mm})$ and large $(\geq 5 \mathrm{~mm})$ in diameter [14].

For risk stratification, additionally to EV size, identification of red spot signs and calculation of Child-Pugh score are crucial, since these endoscopic findings and the Child-Pugh score influence the risk of bleeding and bleeding-related mortality [14, 15]. Importantly, EV can be classified as low-risk $(<5 \mathrm{~mm}$ without endoscopic risk factors in patients with Child-Pugh A/B) or high-risk (all EV of $\geq 5 \mathrm{~mm}$ in diameter or small EV showing red spot signs or small $\mathrm{EV}$ in patients with Child-Pugh $\mathrm{C}$ ) varices regarding the respective risk for variceal bleeding $[14,16]$. Additionally, number of varices and localization should be thoroughly described. Due to the lack of sufficient endoscopic measurement systems, size assessment and endoscopic treatment of EV demand high level of experience and should therefore be performed in specialized centers.

\section{Non-invasive Screening for Any Esophageal Varices and "Varices Needing Treatment" (Table 1)}

Liver stiffness measured by transient elastography (TE) is a well-validated tool for the assessment of liver fibrosis [39]. Recently, the term "compensated advanced chronic liver disease" (cACLD) has been introduced by the Baveno VI consortium [3•] with liver stiffness measurement (LSM) values $>15 \mathrm{kPa}$ being highly suggestive of cACLD. In clinical practice, a qualitative assessment of EV (size as well as presence or absence of red spot signs) is helpful to determine the optimal treatment strategy. Therefore, the term "varices needing treatment (VNT)" has been defined as follows: Varices of medium/large size ( $>5$-mm diameter), or small varices with red spot signs [3•]. According to the Baveno VI criteria, patients with TE values $<20 \mathrm{kPa}$ and a platelet count $\geq 150 \mathrm{G} / \mathrm{L}$ have a very low probability for the presence of VNT. Thus, the combined TE $<20 \mathrm{kPa}$ and platelet count $>$ $150 \mathrm{G} / \mathrm{L}$ can be used to avoid futile screening endoscopies in patients with cACLD [3•].

Multiple studies have evaluated the diagnostic performance of TE alone and/or in combination with other markers (most commonly platelet count or spleen size) to predict the presence/absence of EV and VNT (see Table 1).

Regarding the detection of the presence of varices of any size using TE alone, the proposed cutoffs vary between $13.9 \mathrm{kPa}$ [18] and $29.7 \mathrm{kPa}$ [40]. The corresponding positive predictive values (PPV) ranged from $26 \%$ [37] to $100 \%$ [32] while the negative predictive values (NPV) varied between $31 \%$ [24] and 94\% [36], depending on whether ruling in or ruling out EV was the main aim of the study. TE cutoffs for predicting VNT were usually higher, ranging from $19.0 \mathrm{kPa}$ [18] to $64.5 \mathrm{kPa}$ [41] with corresponding PPV of 25-90\% [19, 41] and NPV of 55-100\% [19, 26, 42] respectively.

By applying the Baveno VI criteria to rule in or rule out the presence of small varices or the "extended Baveno VI criteria" by Augustin et al. [43], small varices would be missed in a considerable number of patients. Only few studies assessed the performance of the Baveno VI criteria to screen for any EV and found a considerably low specificity of $38 \%$ and PPV of $21-43 \%$, but reasonable sensitivity of $88 \%$ and NPV of $86 \%[34,36]$ which is still inferior to the NPV of $97-100 \%$ reported for VNT [36, 44, 45].

Using LSM, low cutoffs, such as the one proposed by Kazemi et al. [18] at $13.9 \mathrm{kPa}$ and validated by Castera et al. in 2009 [21], appear to be more suitable for ruling out small varices with a sensitivity of $92-96 \%$ and a NPV of $85-94 \%$ for varices of all sizes. In a small study including 60 cirrhotic patients with viral hepatitis and/or alcoholic liver disease, Bintintan et al. [32] reported a sensitivity of $95 \%$, a specificity of $100 \%$, a PPV of $100 \%$, and a NPV of $86 \%$ using a LSM cutoff at $15 \mathrm{kPa}$ for screening for varices of any size. The only other study reaching similar values with regard to accuracy 


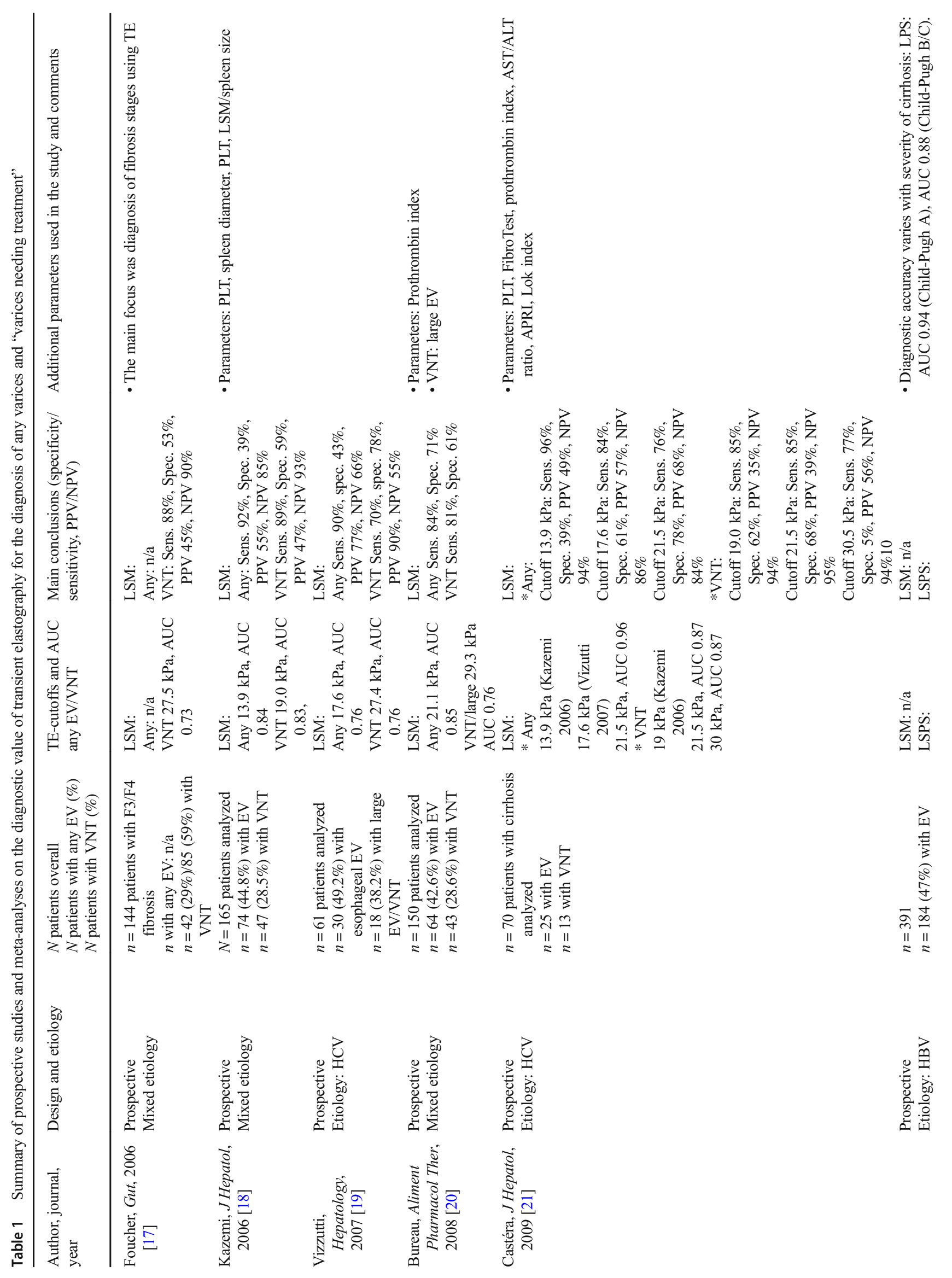




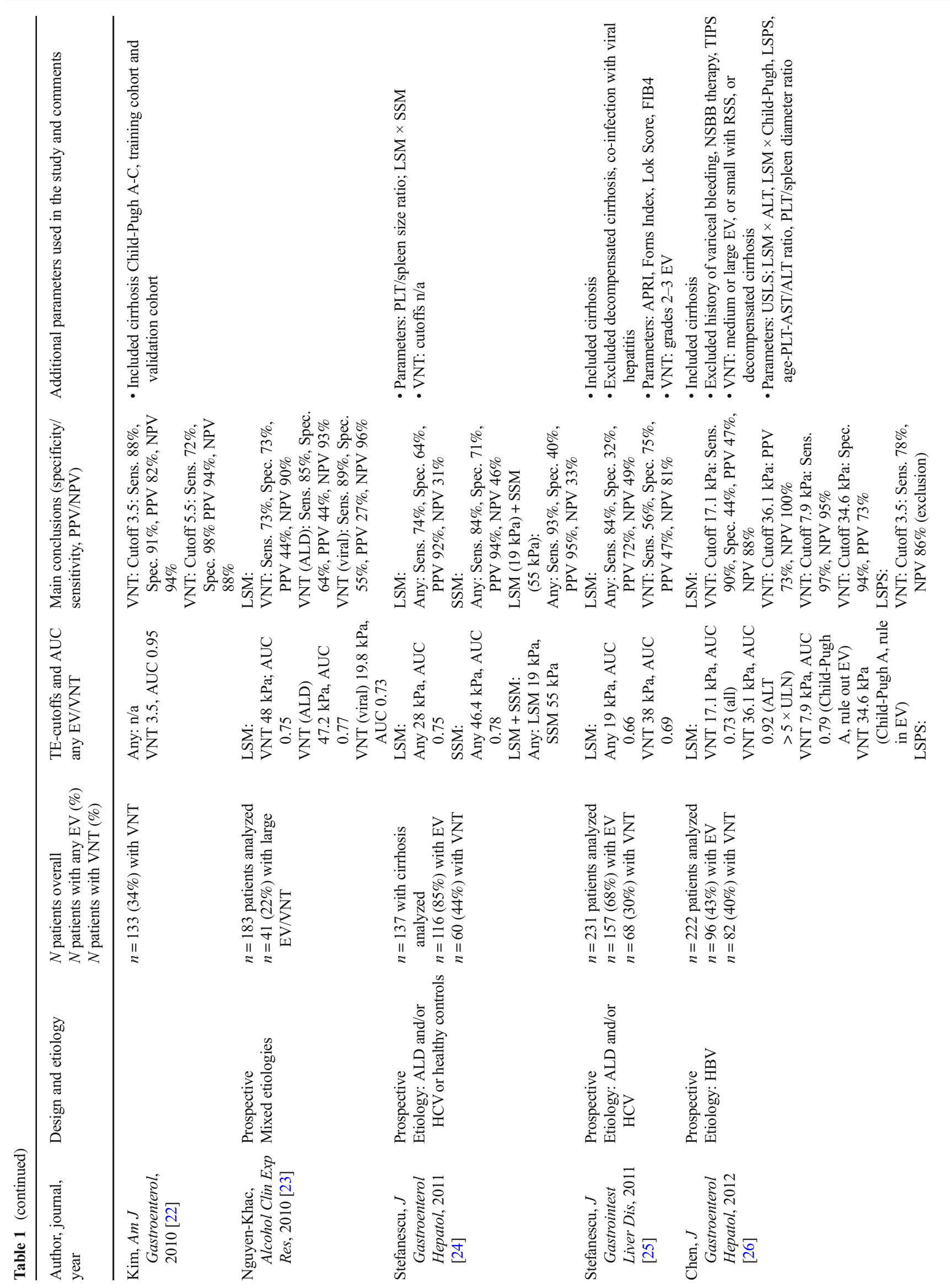




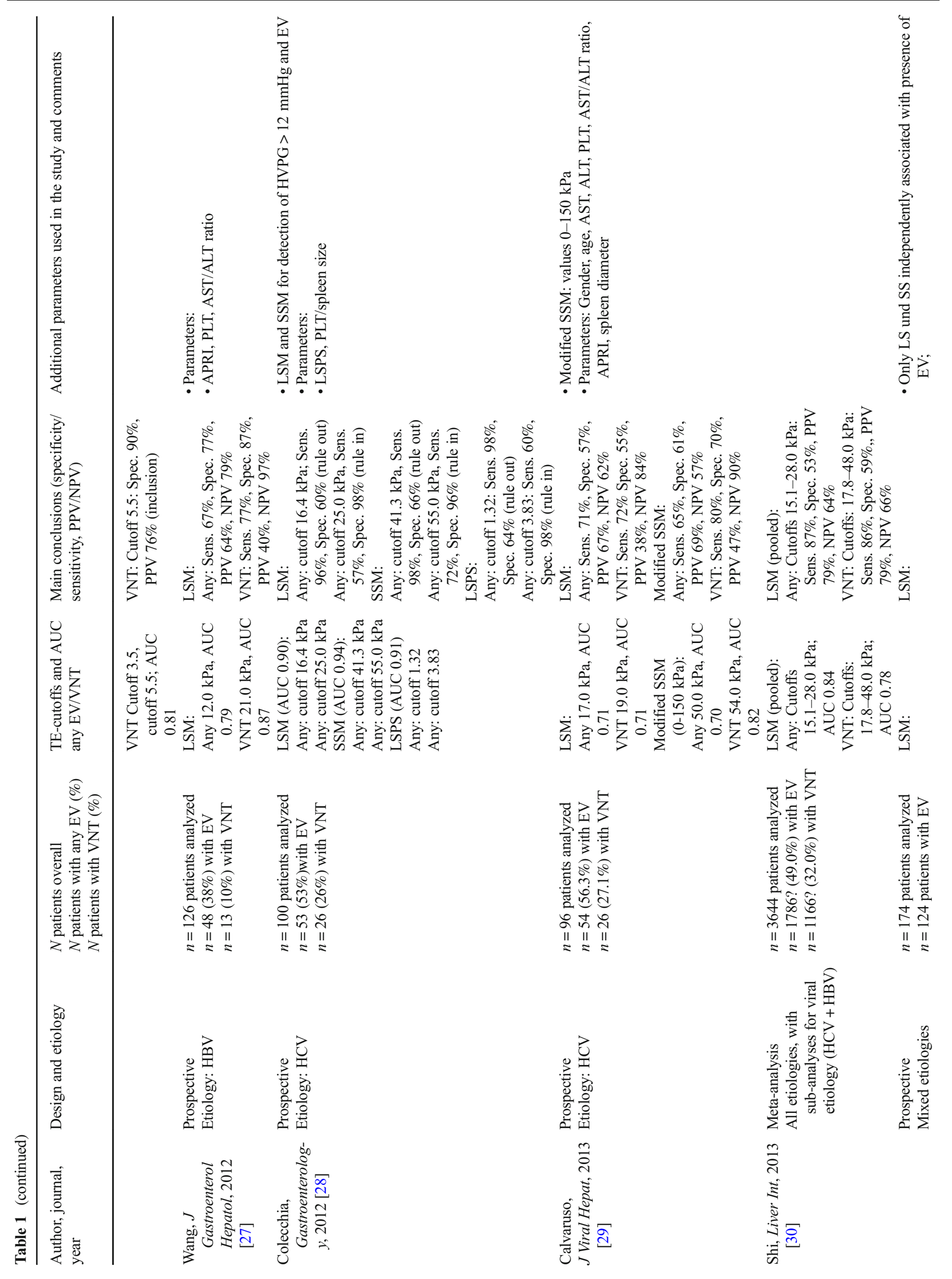




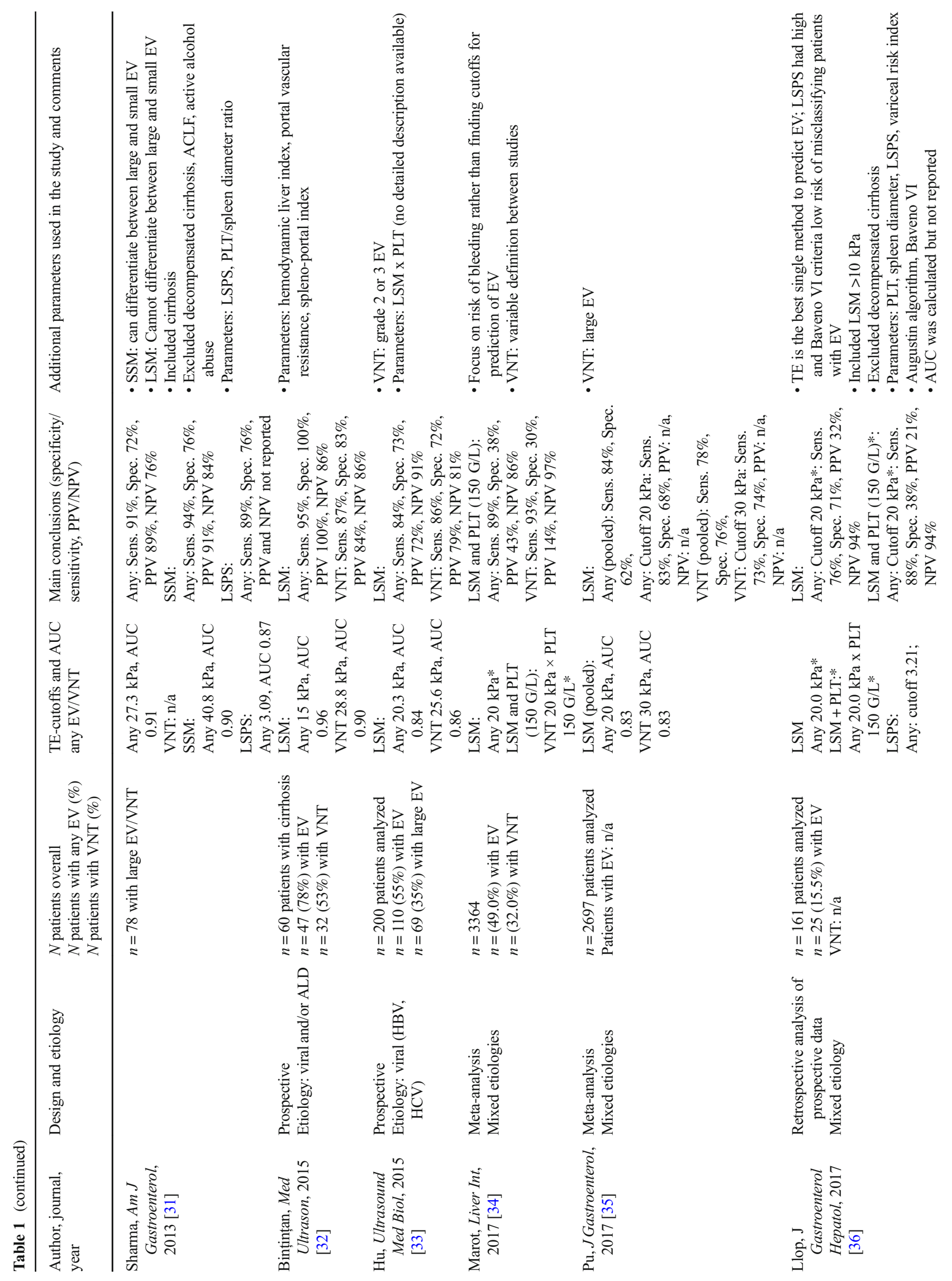




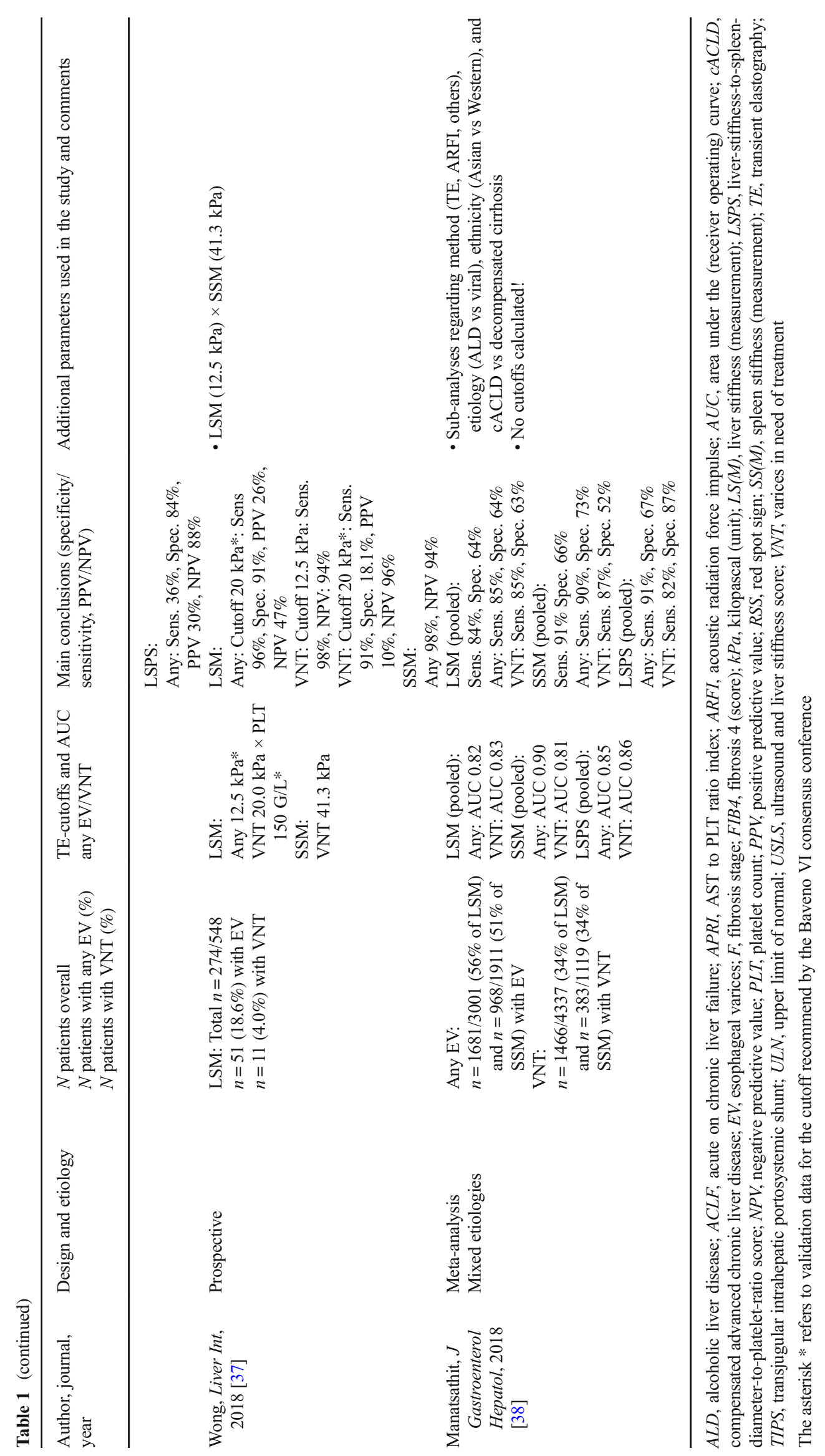


was performed by Stefanescu et al., who used a combination of LSM (cutoff $19 \mathrm{kPa}$ ) and spleen stiffness (SSM, cutoff $55 \mathrm{kPa}$ ) to rule in varices of any size with a sensibility of 93\% and a PPV of 95\% [24].

In summary, LSM using TE in combination with platelet count (i.e., Baveno VI criteria) and/or other non-invasive markers of portal hypertension can be used for ruling out VNT, and thus, avoid unnecessary screening endoscopies in settings where low-risk varices are left untreated. Whether yearly re-evaluation of TE and platelet count is feasible for clinical surveillance and which changes of LSM or SSM or platelet count should call for endoscopic screening must be answered in future studies. As of now, we advise performing endoscopy at any given relevant increase in LSM/SSM or decrease in platelet count as advised by the Baveno VI consensus conference in 2015 [3•]. Importantly, the Baveno VI criteria are not sufficient to rule out small varices/any EV, in clinical practice. Thus, at present, endoscopy is essential if our recommendation to implement medical treatment in all patients with low-risk varices is prefered.

\section{Natural History of Small Varices and Incidence of Bleeding}

An important prospective "natural history" study evaluated the incidence of small varices in 113 patients with cirrhosis of different etiologies without varices at baseline endoscopy and found an incidence rate of 5\% (0.8-8.2\%) and 28\% (21.0$35.0 \%)$ at 1 and 3 years, respectively [9]. Interestingly, only $2 \%(0.1-4.1 \%)$ of patients without varices at first endoscopy bled within 2 years. Among 93 with small EV at baseline, the rate of progression to large EV was $12 \%(5.6-18.4 \%)$ and $31 \%(21.2-40.8 \%)$ at 1 and 3 years, respectively. Alcoholic etiology of liver disease, advanced liver dysfunction (ChildPugh $\mathrm{B} / \mathrm{C}$ ), and red spots signs were all risk factors for progression from small to large varices [9]. Bleeding at 2 years occurred in 12\% (5.2-18.8\%) and the presence of red spot signs at first endoscopy was identified as independent risk factors for variceal bleeding in patients with small varices at baseline [9].

\section{Pathophysiological Considerations Supporting Current Recommendations for the Management of Portal Hypertension/Patients with Small Varices}

Liver fibrosis/cirrhosis increases intrahepatic vascular resistance, and thus, portal pressure [46]. Increases in portal pressure, that is indirectly assessed by the measurement of HVPG, to values of $\geq 10 \mathrm{mmHg}$ denote CSPH [47, 48•]. CSPH is associated with a substantially increased risk for developing EV and/or decompensating events, such as variceal bleeding as well as ascites and its complications. The diagnosis of CSPH is established by HVPG measurement; however, the presence of collaterals such as EV also indicates CSPH.
Thus, in clinical practice, $\mathrm{CSPH}$ is most commonly diagnosed by endoscopy (e.g., the finding of small varices), as HVPG measurement is mostly restricted to academic centers.

Since portal hypertension drives the development of EV and hepatic decompensation, lowering HVPG by NSBB treatment provides a clinically relevant benefit $[47,48 \bullet]$. However, a landmark study by Groszmann and Garcia-Tsao et al. [49], in which patients with cirrhosis and portal hypertension (defined by a hepatic venous pressure gradient $[\mathrm{HVPG}] \geq$ $6 \mathrm{mmHg}$ ) but without varices were randomized to timolol or placebo, demonstrated that NSBB therapy is not generally effective to prevent the occurrence of EV [49]. Although this might seem contradictory, the increasing knowledge on the pathophysiology of portal hypertension and EV development facilitates the interpretation of the findings of this study [50]. CSPH usually develops prior to the occurrence of small EV, in turn, almost every patient with EV has already developed CSPH. While the initial trigger for CSPH development is an increase in intrahepatic vascular resistance, further increases in portal pressure/HVPG are driven by splanchnic vasodilation and hyperdynamic circulation. Since the latter two mechanisms are the targets of conventional NSBB (propranolol, nadolol, or timolol), it becomes evident that most patients without $\mathrm{EV}$, who have a low likelihood of $\mathrm{CSPH}$, do not benefit from NSBB [51・•]. These pathophysiological considerations are also reflected by the "window hypothesis" by Krag et al. [52]. In turn, patients with CSPH who have not yet developed EV might also benefit from NSBB therapy if HVPG can be decreased by $>10 \%$ or to absolute values < $10 \mathrm{mmHg}$ [49].

\section{Impact of Etiological Treatment on Small Varices}

The importance of curing the underlying liver disease was most evident from studies assessing the clinical course of compensated cirrhotic patients with viral hepatitis B and C after successful antiviral treatment in comparison to patients with virological treatment failure.

Lee et al. have reported two cases of patients with HCVrelated cirrhosis, who showed complete regression of their esophageal varices (and splenomegaly) 3 and 8 years after sustained virological response [53]. A prospective observational study assessed the course of EV in patients with versus without sustained virological response (SVR) to interferon/ ribavirin treatment in patients with compensated HCVrelated cirrhosis [54]. Sixty-two patients with SVR and 65 patients without SVR were endoscopically followed for a median of about 5 years ( 68 months vs. 57 months, respectively). Significantly less patients with SVR developed de novo EV (3.5\% vs. $15.1 \%$ without SVR), but SVR only nonsignificantly decreased the progression from small to large EV. Since some patients with SVR developed de novo EV or had progression to large $\mathrm{EV}$, the authors concluded that 
continued endoscopical surveillance for EV is still necessary in patients with HCV cirrhosis despite SVR [9].

Another case of complete regression of EV after suppression of hepatitis B virus (HBV) replication with entecavir has been reported by Jwa et al. [55]. The beneficial impact of long-term nucleos(t)ide analogues (NA) treatment on patients with small EV has been elegantly demonstrated in a prospective, 12-year multicenter study [56••]. Among the 107 $\mathrm{HBeAg}(-)$ patients with compensated cirrhosis, 27 patients had small EV at baseline. Long-term suppression of HBV replication by NA resulted in regression of small $\mathrm{EV}$ in most cases $(83 \%$ cumulative incidence of regression over 12 years, 18/27) and no single patient experienced variceal bleeding. The risk of progression from small to large EV was negligible and occurred almost exclusively in patients with virological breakthrough under NA treatment [56••].

In contrast to patients with viral hepatitis, there are currently no specific therapies approved for alcoholic liver disease (ALD) or non-alcoholic steatohepatitis (NASH). In ALD, strict alcohol abstinence and supervised lifestyle modifications represent critical elements of patient management [57, 58]. Up to date, there are no studies that specifically focus on the impact of alcohol consumption/cessation on the progression of small varices. However, it is well-established that even moderate alcohol consumption worsens portal hypertension and thereby most likely affects the formation and growth of varices. After alcohol cessation, an immediate decrease of HVPG can be observed, which might induce regression of varices [59]. Moreover, Villanueva et al. showed that alcohol cessation improves the hemodynamic response in secondary prophylaxis [60]. In addition, durable alcohol abstinence results in lower re-bleeding rates [61] and decreased long-term mortality after acute variceal bleeding [62]. Negative effects of ongoing alcohol consumption seem to result from increased intrahepatic resistance, due to hepatic inflammation, characterized by parenchymal lesions (hepatocellular ballooning) that are frequently found in active drinkers [63]. In conclusion, alcohol abstinence reduces portal pressure and improves the clinical couse of cirrhotic patients and should therefore be advised to all patients with small varices.

While NASH has become a highly prevalent liver disease [64], there are currently no approved pharmacological therapies for patients with NASH. The "Million Women Study," performed in the UK, suggested that up to $17 \%$ of incident cases of cirrhosis can be directly linked to obesity [65]. Obesity in combination with cirrhosis increases risk of hepatic decompensation and negatively impacts portal hypertension [66]. The pathophysiological mechanisms are still unknown and certainly multifactorial. However, it seems that a persistent proinflammatory state triggers a fibrogenic and angiogenic response in the liver, thereby increasing intrahepatic resistance. In NASH, an increase in portal pressure has been reported in the absence of significant fibrosis
[67] with hepatocellular ballooning due to lipotoxicity and/or only mild perisinusoidal fibrosis [9]. Lifestyle interventions remain the key determinants of NASH progression/regression: Weight loss is associated with decreases in HVPG irrespectively of etiology [68*0]. Importantly, beneficial effects of weight loss may be observed even in the absence of changes in Child-Pugh or MELD score [66, 68••]. In selected cases, bariatric surgery might be beneficial in NASH patients [69].

\section{Treatment of Small Varices with Non-selective Betablockers (Table 2)}

Based on a broad body of evidence, current guidelines recommend either NSBB or EBL for primary prophylaxis of variceal bleeding in patients with medium to large varices. Moreover, NSBB are considered as the mainstay of the combination therapy with EBL therapy in secondary prophylaxis $[5,79]$. However, in patients with small varices who have not bled, the situation is less clear $[3 \cdot, 6,80]$.

In general, there is no conclusive evidence that NSBB treatment reduces the risk of variceal bleeding or mortality in patients with low-risk varices who have not bled [5]. However, most trials were not sufficiently powered to detect favorable effects on these endpoints, which occur less commonly in this group of patients [6]. This is the reason why well-designed studies on clinically meaningful surrogate endpoints (e.g., variceal growth) are particularly relevant in this setting.

While a randomized controlled trial (RCT) by Merkel et al. [7] demonstrated that conventional NSBB (nadolol) therapy is effective in preventing the progression from small to large varices in patients who have not bled, another RCT by Sarin and co-workers [8] using propranolol reported no effect. In a recently published randomized RCT by Bhardwaj and colleagues [77], a lower proportion of patients assigned to the carvedilol group progressed from small to large varices as compared to the placebo group. Carvedilol has been shown to be more potent in decreasing portal pressure due to its additional anti- $\alpha 1$-adrenergic activity [81]. In a recent metaanalysis [82], the mean relative HVPG reduction was $22 \%$ for carvedilol and $16 \%$ for propranolol, resulting in a weighed mean difference of $7 \%$ in favor of carvedilol. However, owing to its anti- $\alpha 1$-adrenergic activity [81], carvedilol might also lead to more pronounced decreases in systemic arterial pressure, when compared to conventional NSBB, which limits its use in patients with severe or refractory ascites [5, 79॰].

We recently conducted a meta-analysis of RCT on NSBB treatment restricted to patients with small varices, also including the new study by Bhardwaj and co-workers [77]. Interestingly, in our meta-analysis [83], there was a trend towards an amelioration of the progression from small to large varices among NSBB-treated patients in the fixed effects model (odds ratio [OR] [95\% confidence interval $(95 \% \mathrm{CI})]$ 0.73 [0.5-1.06]; risk ratio [RR] [95\% CI] 0.78 [0.58-1.05]). 


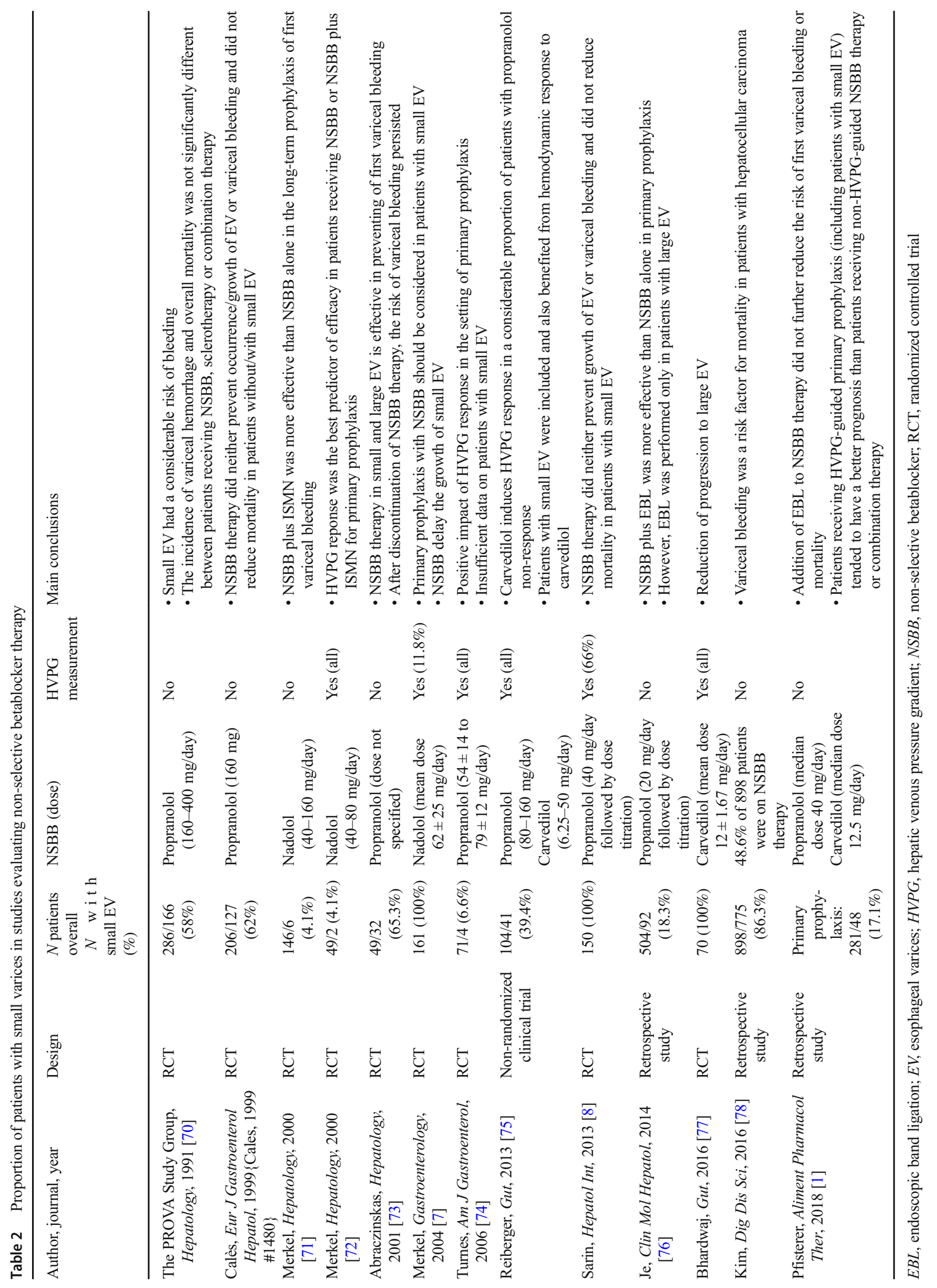


However, the random effects model might have been more accurate due to study heterogeneity. In this model, the $95 \%$ CI was substantially wider (OR [95\% CI] 0.76 [0.25 to 2.29]; RR [95\% CI] 0.82 [0.36 to 1.87]). Thus, the findings of our meta-analysis [83] plead for further studies to confirm the effectiveness of NSBB therapy (especially carvedilol) for preventing variceal growth.

\section{The Role of Hepatic Venous Pressure Gradient in Guiding Treatment Decisions in Patients with Small Varices}

Considering varices of all size (i.e., also medium to large varices), NSBB treatment reduces the 2-year risk of first variceal bleeding from 25 to $15 \%$, resulting in a number needed to treat [NNT] of 10 [84]. In the group of patients with small varices, however, the NNT for preventing variceal bleeding might be considerably higher (about 20), underlining the importance of identifying patients who are most likely to benefit. To date, HVPG response is the only established surrogate for the effectiveness of NSBB therapy. Due to the need for two separate HVPG measurements, the evaluation of "chronic" HVPG response to NSBB treatment is very resource-intensive. In contrast, the assessment of "acute" HVPG response to i.v. propranolol is performed in a single session, and thus, provides a valuable alternative $[85,86]$. For primary prophylaxis, the Baveno VI faculty recently unified the definition of HVPG response by using the same criteria (HVPG decrease $\geq$ $10 \%$ or to a value of $\leq 12 \mathrm{mmHg}$ ) for "acute" and "chronic" assessments [3•]. Although most of the evidence for the impact of a decrease in HVPG on hepatic decompensation [87] and mortality [88] is derived from studies comprising patients with medium-large varices, there are also some studies focusing on patients with less advanced disease. In a landmark study [49] assigning patients with portal hypertension who had not developed varices (pre-primary prophylaxis) to timolol or placebo, patients who had a relative HVPG decrease of $>10 \%$ after 1 year showed a reduced incidence of the composite primary endpoint (development of varices or variceal bleeding) [49]. Moreover, a recent RCT evaluated the impact of HVPG-guided therapy on hepatic decompensation in patients with clinically significant portal hypertension $(\mathrm{CSPH}$; pre-primary prophylaxis [44\%] or small varices without red spot signs [56\%]). Two hundred one patients were randomized to NSBB therapy (propranolol or carvedilol in patients with and without "acute" HVPG response to i.v. propranolol, respectively) or placebo. Interestingly, the rates of hepatic decompensation were $16 \%$ and $27 \%$ in the NSBB and placebo group, respectively. Thus, HVPG-guided therapy with propranolol/carvedilol substantially reduced the risk of hepatic decompensation (hazard ratio [95\% CI] 0.51 [0.58 to 1.05]), primarily by decreasing the incidence of ascites [89••], which is the most frequent first decompensating event [90].
Since nearly all patients with cirrhosis and small varices have $\mathrm{CSPH}$, the findings of the aforementioned study [89••], as well as the potential effect of NSBB treatment on the progression of varices, provide a good rationale for HVPGguided NSBB therapy in patients with small varices. This approach allows to identify patients who are likely to benefit from propranolol, while hemodynamic non-responders can be treated with carvedilol, which still achieves HVPG response in a significant proportion of these patients [69]. Moreover, information on HVPG response might facilitate treatment individualization in situations in which NSBB might have a less favorable safety profile $[5,79 \bullet]$, e.g., patients with refractory ascites [91], spontaneous bacterial peritonitis [92], or severe alcoholic hepatitis [93]. If HVPG measurement is not available, carvedilol $12.5 \mathrm{mg}$ q.d. might be the NSBB of choice in patients without severe ascites.

\section{Our Personal Recommendations for Screening and Management of Small Varices (Fig. 1)}

Here, we want to provide the reader with recommendations for daily clinical practice, that - in absence of RCT on some specific aspects - may not always be based on high-quality evidence. First, TE cutoffs $<20 \mathrm{kPa}$ applied to patients with cACLD even combined with platelet count $>150 \mathrm{G} / \mathrm{L}$ cannot sufficiently rule out the presence of small varices. Accordingly, endoscopy is essential if low-risk varices are intended to be treated with pharmacological therapy. If liver stiffness is measured at $\geq 20 \mathrm{kPa}$ and/or platelet count is $<150 \mathrm{G} / \mathrm{L}$, screening endoscopy must be performed regardless of the treatment strategy. Moreover, the occurrence of hepatic decompensation should prompt endoscopy.

Varices should then be classified as absent, small $(<5 \mathrm{~mm})$, or large $(\geq 5 \mathrm{~mm})$. Further, presence of red spot signs and Child-Pugh score needs to be evaluated for risk stratification.

If small varices are detected, in addition to etiological therapy, we initiate NSBB therapy due to its potential beneficial effects on variceal growth and the reduced risk of hepatic decompensation. Importantly, NSBB seem to be effective even in patients with small (and large) varices who remain strictly abstinent from alcohol $[9,68 \cdot \bullet, 69,73]$ and in patients who receive effective antiviral therapy [68••]. This would support the treatment of small varices both in patients with ongoing hepatic injury and in patients with effective treatment of the underlying etiology. Thus, we use NSBB in all patients with small varices, independently if the etiological factor is eliminated or not.

Acute HVPG response can be evaluated by administration of i.v. propranolol within a single session, while chronic assessment of HVPG response requires a second HVPG measurement around 3-5 weeks after initiation of conventional NSBB/carvedilol therapy. This HVPG-guided approach 


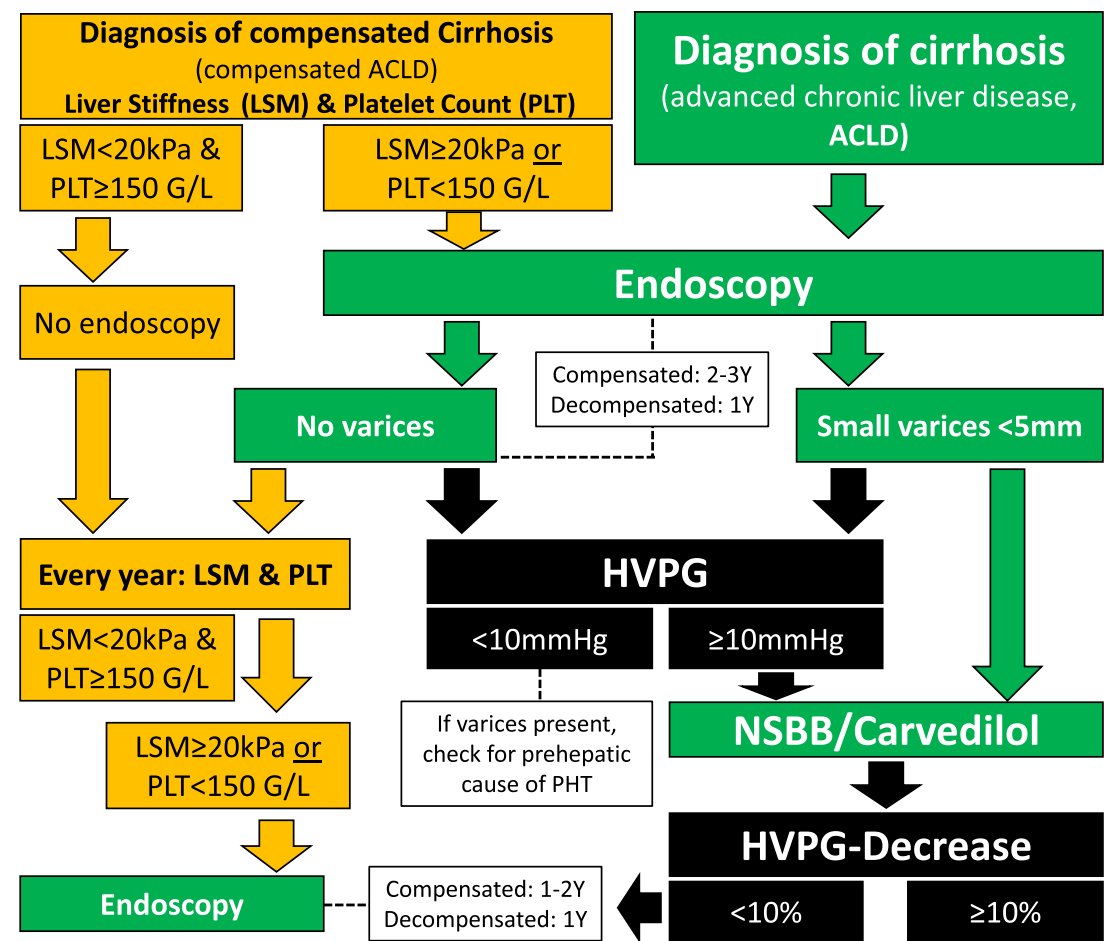

Fig. 1 Suggested algorithm for the diagnosis and management of small varices. The green elements summarize the recommended strategy as supported by previous international guidelines that recommend screening endoscopy in all patients diagnosed with cirrhosis (i.e., ACLD). If small varices are detected, treatment with conventional NSBB or carvedilol may be started as primary prophylaxis of variceal bleeding. If no varices are detected, screening endoscopy should be repeated every 1-2 years. The orange elements summarize the Baveno VI recommendations for non-invasive diagnosis of varices in patients with cirrhosis that were designed to avoid screening endoscopies in patients with low liver stiffness $(\mathrm{TE}<20 \mathrm{kPa})$ and normal platelet counts (PLT $\geq 150 \mathrm{G} / \mathrm{L}$ ). However, since this strategy will miss a considerable number of patients with small varices (that must not be treated-but should be treated in our opinion), we would still recommend to perform screening endoscopies even in patients with

allows to identify patients who are likely to benefit from propranolol, while hemodynamic non-responders can be treated with carvedilol, which still achieves chronic HVPG response in a significant proportion of these patients. A decrease in HVPG $\geq 10 \%$ or to a value of $\leq 12 \mathrm{mmHg}$ defines NSBB response. If HVPG measurement is not available, we recommend the use of carvedilol $12.5 \mathrm{mg}$ q.d. in patients without severe ascites.

In general, we would also recommend NSBB treatment for patients with $\mathrm{CSPH}$ but without EV, since HVPG response may prevent the development of varices as well as decompensating events in compensated patients.

In patients with severe/refractory ascites, arterial blood pressure should be monitored since NSBB might compromise the circulatory reserve. The risk-benefit ratio/dosing of NSBB treatment should be re-evaluated in patients with arterial hypotension, spontaneous bacterial peritonitis, and/or acute kidney injury. In some patients, NSBB therapy might need to be
TE $\geq 20 \mathrm{kPa}$ or PLT $<150 \mathrm{G} / \mathrm{L}$ if cirrhosis (ACLD) is suspected. Finally, the black elements indicate advanced diagnostic/therapeutic options that we recommend for optimal management of patients with small varices: In patients with small varices and even in patients with ACLD without varices (especially in those with TE $\geq 20 \mathrm{kPa}$ or PLT $<$ $150 \mathrm{G} / \mathrm{L}$ ), HVPG should be measured. If HVPG is measured at $\geq$ $10 \mathrm{mmHg}$, the hemodynamic response to NSBB (ideally intravenous testing) or carvedilol should be assessed and hemodynamic responders should be kept on NSBB or carvedilol if a decrease of at least $\geq 10 \%$ in HVPG is observed. In hemodynamic non-responders, follow-up endoscopy should be performed every 1-2 years in order to assess the progression to large varices. ACLD, advanced chronic liver disease; cACLD compensated advanced chronic liver disease; HVPG, hepatic venous pressure gradient; LSM, liver stiffness measurement; NSBB, non-selective betablocker; PLT, platelet count; Y, year(s)

discontinued if arterial hypotension is severe or shock/AKI requiring vasopressors occurs; however, this might be indicative of worse prognosis and these patients should then be evaluated for more aggressive treatment strategies such as TIPS or liver transplantation.

\section{Conclusions}

The main therapeutic focus in cirrhotic patients with small varices ( $\leq 5 \mathrm{~mm}$ in diameter) is the cure of the underlying etiology. The optimal management of small varices should include measurement of HVPG. A pharmacological decrease in HVPG by NSBB therapy $\geq 10 \%$ reduces the risk of progression to large varices, first variceal bleeding, and hepatic decompensation. If HVPG is not available, we would recommend carvedilol $12.5 \mathrm{mg}$ once daily for treatment of small varices in patients without severe ascites. Only if small EV 
are not treated or in hemodynamic non-responders, follow-up endoscopies should be performed in 1-2 yearly intervals considering the activity of liver disease or if hepatic decompensation occurs.

Funding Information Open access funding provided by Medical University of Vienna.

\section{Compliance with Ethical Standards}

Conflict of Interest Thomas Reiberger, Theresa Bucsics, Rafael Paternostro, Nikolaus Pfisterer, Florian Riedl, and Mattias Mandorfer each declare no potential conflict of interest.

Human and Animal Rights and Informed Consent All reported studies/ experiments with human or animal subjects performed by the authors have been previously published and complied with all applicable ethical standards (including the Helsinki declaration and its amendments, institutional/national research committee standards, and international/national/institutional guidelines).

Open Access This article is distributed under the terms of the Creative Commons Attribution 4.0 International License (http:// creativecommons.org/licenses/by/4.0/), which permits unrestricted use, distribution, and reproduction in any medium, provided you give appropriate credit to the original author(s) and the source, provide a link to the Creative Commons license, and indicate if changes were made.

\section{References}

Papers of particular interest, published recently, have been highlighted as:

- Of importance

-• Of major importance

1. Pfisterer N, Dexheimer C, Fuchs EM, Bucsics T, Schwabl P, Mandorfer M, et al. Betablockers do not increase efficacy of band ligation in primary prophylaxis but they improve survival in secondary prophylaxis of variceal bleeding. Aliment Pharmacol Ther. 2018;47(7):966-79.

2. Albillos A, Zamora J, Martinez J, et al. Stratifying risk in the prevention of recurrent variceal hemorrhage: results of an individual patient meta-analysis. Hepatology. 2017;66(4):1219-31.

3. de Franchis R, Baveno VIF. Expanding consensus in portal hypertension: report of the Baveno VI Consensus Workshop: stratifying risk and individualizing care for portal hypertension. J Hepatol. 2015;63(3):743-52 The most recent European guidelines for the management of patients with cirrhosis and portal hypertension.

4. Garcia-Tsao G, Abraldes JG, Berzigotti A, Bosch J. Portal hypertensive bleeding in cirrhosis: risk stratification, diagnosis, and management: 2016 practice guidance by the American Association for the study of liver diseases. Hepatology. 2017;65(1):310-35 The most recent American guidelines for the management of patients with cirrhosis and portal hypertension.

5. Mandorfer M, Reiberger T. Beta blockers and cirrhosis, 2016. Dig Liver Dis. 2017;49(1):3-10.

6. Tripathi D, Stanley AJ, Hayes PC, Patch D, Millson C, Mehrzad H, et al. U.K. guidelines on the management of variceal haemorrhage in cirrhotic patients. Gut. 2015;64(11):1680-704.
7. Merkel C, Marin R, Angeli P, Zanella P, Felder M, Bernardinello E, et al. A placebo-controlled clinical trial of nadolol in the prophylaxis of growth of small esophageal varices in cirrhosis. Gastroenterology. 2004;127(2):476-84.

8. Sarin SK, Mishra SR, Sharma P, Sharma BC, Kumar A. Early primary prophylaxis with beta-blockers does not prevent the growth of small esophageal varices in cirrhosis: a randomized controlled trial. Hepatol Int. 2013;7(1):248-56.

9. Merli M, Nicolini G, Angeloni S, Rinaldi V, de Santis A, Merkel C, et al. Incidence and natural history of small esophageal varices in cirrhotic patients. J Hepatol. 2003;38(3):266-72.

10. Brick IB, Palmer ED. One thousand cases of portal cirrhosis of the liver. Implications of esophageal varices and their management. Arch Intern Med. 1964;113:501-11.

11. Dagradi AE, Stempien SJ, Owens LK. Bleeding esophagogastric varices. An endoscopic study of 50 cases. Arch Surg. 1966;92(6):944-7.

12. The general rules for recording endoscopic findings on esophageal varices. Jpn J Surg. 1980;10(1):84-7.

13. Paquet KJ. Prophylactic endoscopic sclerosing treatment of the esophageal wall in varices - a prospective controlled randomized trial. Endoscopy. 1982;14(1):4-5.

14. Reiberger T, Puspok A, Schoder M, et al. Austrian consensus guidelines on the management and treatment of portal hypertension (Billroth III). Wien Klin Wochenschr. 2017;129(Suppl 3):135-58.

15. Bosch J, Abraldes JG, Groszmann R. Current management of portal hypertension. J Hepatol. 2003;38(Suppl 1):S54-68.

16. North Italian Endoscopic Club for the S, Treatment of Esophageal V. Prediction of the first variceal hemorrhage in patients with cirrhosis of the liver and esophageal varices. A prospective multicenter study. N Engl J Med. 1988;319(15):983-9.

17. Foucher J, Chanteloup E, Vergniol J, Castéra L, le Bail B, Adhoute $\mathrm{X}$, et al. Diagnosis of cirrhosis by transient elastography (FibroScan): a prospective study. Gut. 2006;55(3):403-8.

18. Kazemi F, Kettaneh A, N'Kontchou G, et al. Liver stiffness measurement selects patients with cirrhosis at risk of bearing large oesophageal varices. J Hepatol. 2006;45(2):230-5.

19. Vizzutti F, Arena U, Romanelli RG, Rega L, Foschi M, Colagrande $\mathrm{S}$, et al. Liver stiffness measurement predicts severe portal hypertension in patients with HCV-related cirrhosis. Hepatology. 2007;45(5):1290-7.

20. Bureau C, Metivier S, Peron JM, et al. Transient elastography accurately predicts presence of significant portal hypertension in patients with chronic liver disease. Aliment Pharmacol Ther. 2008;27(12):1261-8.

21. Castera L, Le Bail B, Roudot-Thoraval F, et al. Early detection in routine clinical practice of cirrhosis and oesophageal varices in chronic hepatitis C: comparison of transient elastography (FibroScan) with standard laboratory tests and non-invasive scores. J Hepatol. 2009;50(1):59-68.

22. Kim BK, Han KH, Park JY, Ahn SH, Kim JK, Paik YH, et al. A liver stiffness measurement-based, noninvasive prediction model for high-risk esophageal varices in B-viral liver cirrhosis. Am J Gastroenterol. 2010;105(6):1382-90.

23. Nguyen-Khac E, Saint-Leger P, Tramier B, Coevoet H, Capron D, Dupas JL. Noninvasive diagnosis of large esophageal varices by Fibroscan: strong influence of the cirrhosis etiology. Alcohol Clin Exp Res. 2010;34(7):1146-53.

24. Stefanescu H, Grigorescu M, Lupsor M, Procopet B, Maniu A, Badea R. Spleen stiffness measurement using Fibroscan for the noninvasive assessment of esophageal varices in liver cirrhosis patients. J Gastroenterol Hepatol. 2011;26(1):164-70.

25. Stefanescu H, Grigorescu M, Lupsor M, Maniu A, Crisan D, Procopet B, et al. A new and simple algorithm for the noninvasive assessment of esophageal varices in cirrhotic patients using serum fibrosis markers and transient elastography. J Gastrointest Liver Dis: JGLD. 2011;20(1):57-64. 
26. Chen YP, Zhang Q, Dai L, Liang XE, Peng J, Hou JL. Is transient elastography valuable for high-risk esophageal varices prediction in patients with hepatitis-B-related cirrhosis? J Gastroenterol Hepatol. 2012;27(3):533-9.

27. Wang JH, Chuah SK, Lu SN, Hung CH, Chen $\mathrm{CH}$, Kee KM, et al. Transient elastography and simple blood markers in the diagnosis of esophageal varices for compensated patients with hepatitis B virusrelated cirrhosis. J Gastroenterol Hepatol. 2012;27(7):1213-8.

28. Colecchia A, Montrone L, Scaioli E, Bacchi-Reggiani ML, Colli A, Casazza G, et al. Measurement of spleen stiffness to evaluate portal hypertension and the presence of esophageal varices in patients with HCV-related cirrhosis. Gastroenterology. 2012;143(3):646-54.

29. Calvaruso V, Bronte F, Conte E, Simone F, Craxi A, Di Marco V. Modified spleen stiffness measurement by transient elastography is associated with presence of large oesophageal varices in patients with compensated hepatitis C virus cirrhosis. J Viral Hepat. 2013;20(12):867-74.

30. Shi KQ, Fan YC, Pan ZZ, Lin XF, Liu WY, Chen YP, et al. Transient elastography: a meta-analysis of diagnostic accuracy in evaluation of portal hypertension in chronic liver disease. Liver Int. 2013;33(1):62-71.

31. Sharma P, Kirnake V, Tyagi P, Bansal N, Singla V, Kumar A, et al. Spleen stiffness in patients with cirrhosis in predicting esophageal varices. Am J Gastroenterol. 2013;108(7):1101-7.

32. Bintintan A, Chira RI, Bintintan VV, Nagy G, Manzat-Saplacan RM, Lupsor Platon M, et al. Value of hepatic elastography and Doppler indexes for predictions of esophageal varices in liver cirrhosis. Med Ultrason. 2015;17(1):5-11.

33. Hu Z, Li Y, Li C, Huang C, Ou Z, Guo J, et al. Using ultrasonic transient elastometry (FibroScan) to predict esophageal varices in patients with viral liver cirrhosis. Ultrasound Med Biol. 2015;41(6): 1530-7.

34. Marot A, Trepo E, Doerig C, Schoepfer A, Moreno C, Deltenre P. Liver stiffness and platelet count for identifying patients with compensated liver disease at low risk of variceal bleeding. Liver Int. 2017;37(5):707-16.

35. Pu K, Shi JH, Wang X, Tang Q, Wang XJ, Tang KL, et al. Diagnostic accuracy of transient elastography (FibroScan) in detection of esophageal varices in patients with cirrhosis: a meta-analysis. World J Gastroenterol: WJG. 2017;23(2):345-56.

36. Llop E, Lopez M, de la Revilla J, Fernandez N, Trapero M, Hernandez M, et al. Validation of noninvasive methods to predict the presence of gastroesophageal varices in a cohort of patients with compensated advanced chronic liver disease. J Gastroenterol Hepatol. 2017;32(11):1867-72

37. Wong GLH, Kwok R, Hui AJ, Tse YK, Ho KT, Lo AOS, et al. A new screening strategy for varices by liver and spleen stiffness measurement (LSSM) in cirrhotic patients: a randomized trial. Liver Int. 2018;38(4):636-44.

38. Manatsathit W, Samant H, Kapur S, Ingviya T, Esmadi M, Wijarnpreecha K, et al. Accuracy of liver stiffness, spleen stiffness, and LS-spleen diameter to platelet ratio score in detection of esophageal varices: systemic review and meta-analysis. J Gastroenterol Hepatol. 2018;33:1696-706.

39. Friedrich-Rust M, Ong MF, Martens S, et al. Performance of transient elastography for the staging of liver fibrosis: a meta-analysis. Gastroenterology. 2008;134(4):960-74.

40. Saad Y, Said M, Idris MO, Rabee A, Zakaria S. Liver stiffness measurement by fibroscan predicts the presence and size of esophageal varices in egyptian patients with $\mathrm{HCV}$ related liver cirrhosis. J Clin Diagn Res : JCDR. 2013;7(10):2253-7.

41. Pineda JA, Recio E, Camacho A, Macías J, Almodóvar C, González-Serrano M, et al. Liver stiffness as a predictor of esophageal varices requiring therapy in HIV/hepatitis $C$ virus-coinfected patients with cirrhosis. J Acquir Immune Defic Syndr (1999). 2009;51(4):445-9.
42. Jangouk P, Turco L, De Oliveira A, Schepis F, Villa E, Garcia-Tsao G. Validating, deconstructing and refining Baveno criteria for ruling out high-risk varices in patients with compensated cirrhosis. Liver Int 2017;37(8):1177-1183.

43. Augustin S, Pons M, Maurice JB, et al. Expanding the Baveno VI criteria for the screening of varices in patients with compensated advanced chronic liver disease, Hepatology (Baltimore MD 2017;66(6):1980-1988.

44. Colecchia A, Ravaioli F, Marasco G, Colli A, Dajti E, di Biase AR, et al. A combined model based on spleen stiffness measurement and Baveno VI criteria to rule out high-risk varices in advanced chronic liver disease. J Hepatol. 2018;69:308-17.

45. Maurice JB, Brodkin E, Arnold F, Navaratnam A, Paine H, Khawar $\mathrm{S}$, et al. Validation of the Baveno VI criteria to identify low risk cirrhotic patients not requiring endoscopic surveillance for varices. J Hepatol. 2016;65(5):899-905.

46. Tsochatzis EA, Bosch J, Burroughs AK. Liver cirrhosis. Lancet. 2014;383(9930):1749-61.

47. Abraldes JG, Sarlieve P, Tandon P. Measurement of portal pressure. Clin Liver Dis. 2014;18(4):779-92.

48. La Mura V, Nicolini A, Tosetti G, Primignani M. Cirrhosis and portal hypertension: the importance of risk stratification, the role of hepatic venous pressure gradient measurement. World J Hepatol. 2015;7(4):688-95 Excellent review summarizing the importance of HVPG measurements for guiding the management of cirrhotic patients with portal hypertension.

49. Groszmann RJ, Garcia-Tsao G, Bosch J, Grace ND, Burroughs AK, Planas R, et al. Beta-blockers to prevent gastroesophageal varices in patients with cirrhosis. N Engl J Med. 2005;353(21):2254-61.

50. Bosch J, Groszmann RJ, Shah VH. Evolution in the understanding of the pathophysiological basis of portal hypertension: how changes in paradigm are leading to successful new treatments. J Hepatol. 2015;62(1 Suppl):S121-30.

51.・Villanueva C, Albillos A, Genesca J, et al. Development of hyperdynamic circulation and response to beta-blockers in compensated cirrhosis with portal hypertension. Hepatology. 2015; Excellent study providing evidence that NSBB therapy is more effective after CSPH has developed.

52. Krag A, Wiest R, Albillos A, Gluud LL. The window hypothesis: haemodynamic and non-haemodynamic effects of beta-blockers improve survival of patients with cirrhosis during a window in the disease. Gut. 2012;61(7):967-9.

53. Lee SJ, Cho YK, Na SY, Choi EK, Boo SJ, Jeong SU, et al. Regression of esophageal varices and splenomegaly in two patients with hepatitis-C-related liver cirrhosis after interferon and ribavirin combination therapy. Clin Mol Hepatol. 2016;22(3):390-5.

54. D'Ambrosio R, Aghemo A, Rumi MG, et al. The course of esophageal varices in patients with hepatitis $\mathrm{C}$ cirrhosis responding to interferon/ribavirin therapy. Antivir Ther. 2011;16(5):677-84.

55. Jwa HY, Cho YK, Choi EK, Kim HU, Song HJ, Na SY, et al. Regression of esophageal varices during entecavir treatment in patients with hepatitis-B-virus-related liver cirrhosis. Clin Mol Hepatol. 2016;22(1):183-7.

$56 . \bullet$ Lampertico P, Invernizzi F, Vigano M, et al. The long-term benefits of nucleos $(\mathrm{t})$ ide analogs in compensated HBV cirrhotic patients with no or small esophageal varices: a 12-year prospective cohort study. J Hepatol. 2015;63(5):1118-25 Large prospective study showing the beneficial impact of etiological therapy in patients with small varices.

57. Osna NA, Donohue TM Jr, Kharbanda KK. Alcoholic liver disease: pathogenesis and current management. Alcohol Res : Curr Rev. 2017;38(2):147-61.

58. Addolorato G, Mirijello A, Barrio P, Gual A. Treatment of alcohol use disorders in patients with alcoholic liver disease. J Hepatol. 2016;65(3):618-30. 
59. Klein CP, Kalk JF, Muting D, Klein CG. The effect of alcohol on portal vein hemodynamics in nutritional-toxic liver cirrhosis. Deutsche medizinische Wochenschrift (1946). 1993;118(4):89-93.

60. Villanueva C, Lopez-Balaguer JM, Aracil C, et al. Maintenance of hemodynamic response to treatment for portal hypertension and influence on complications of cirrhosis. J Hepatol. 2004;40(5): 757-65.

61. Muntaner L, Altamirano JT, Augustin S, González A, Esteban R, Guardia J, et al. High doses of beta-blockers and alcohol abstinence improve long-term rebleeding and mortality in cirrhotic patients after an acute variceal bleeding. Liver Int. 2010;30(8):1123-30.

62. Jaurigue MM, Cappell MS. Therapy for alcoholic liver disease. World J Gastroenterol : WJG. 2014;20(9):2143-58.

63. Spahr L, Goossens N, Furrer F, Dupuis M, Vijgen S, Elkrief L, et al. A return to harmful alcohol consumption impacts on portal hemodynamic changes following alcoholic hepatitis. Eur J Gastroenterol Hepatol. 2018;30(8):967-74.

64. Argo CK, Caldwell SH. Epidemiology and natural history of nonalcoholic steatohepatitis. Clin Liver Dis. 2009;13(4):511-31.

65. Liu B, Balkwill A, Reeves G, Beral V. Body mass index and risk of liver cirrhosis in middle aged UK women: prospective study. BMJ (Clinical research ed). 2010;c912:340.

66. Berzigotti A, Abraldes JG. Impact of obesity and insulin-resistance on cirrhosis and portal hypertension. Gastroenterologia y hepatologia. 2013;36(8):527-33.

67. Spengler EK, O'Leary JG, Te HS, et al. Liver transplantation in the obese cirrhotic patient. Transplantation. 2017;101(10):2288-96.

68.• Bhardwaj A, Kedarisetty CK, Vashishtha C, et al. Carvedilol delays the progression of small oesophageal varices in patients with cirrhosis: a randomised placebo-controlled trial. Gut. 2017;66(10): 1838-43 Recent RCT showing that carvedilol inhibits the progression from small to large varices.

69. Reiberger T, Ulbrich G, Ferlitsch A, Payer BA, Schwabl P, Pinter $\mathrm{M}$, et al. Carvedilol for primary prophylaxis of variceal bleeding in cirrhotic patients with haemodynamic non-response to propranolol. Gut. 2013;62(11):1634-41.

70. The PROVA Study Group. Prophylaxis of first hemorrhage from esophageal varices by sclerotherapy, propranolol or both in cirrhotic patients: a randomized multicenter trial. Hepatology. 1991;14(6): 1016-24

71. Merkel C, Marin R, Sacerdoti D, Donada C, Cavallarin G, Torboli $\mathrm{P}$, et al. Long-term results of a clinical trial of nadolol with or without isosorbide mononitrate for primary prophylaxis of variceal bleeding in cirrhosis. Hepatology. 2000;31(2):324-9.

72. Merkel C, Bolognesi M, Sacerdoti D, Bombonato G, Bellini B, Bighin R, et al. The hemodynamic response to medical treatment of portal hypertension as a predictor of clinical effectiveness in the primary prophylaxis of variceal bleeding in cirrhosis. Hepatology. 2000;32(5):930-4.

73. Abraczinskas DR, Ookubo R, Grace ND, Groszmann RJ, Bosch J, Garcia-Tsao G, et al. Propranolol for the prevention of first esophageal variceal hemorrhage: a lifetime commitment? Hepatology. 2001;34(6):1096-102.

74. Turnes J, Garcia-Pagan JC, Abraldes JG, Hernandez-Guerra M, Dell'Era A, Bosch J. Pharmacological reduction of portal pressure and long-term risk of first variceal bleeding in patients with cirrhosis. Am J Gastroenterol. 2006;101(3):506-12.

75. Reiberger T, Ulbrich G, Ferlitsch A, Payer BA, Schwabl P, Pinter $\mathrm{M}$, et al. Carvedilol for primary prophylaxis of variceal bleeding in cirrhotic patients with haemodynamic non-response to propranolol. Gut. 2013;62(11):1634-41

76. Je D, Paik YH, Gwak GY, Choi MS, Lee JH, Koh KC, et al. The comparison of esophageal variceal ligation plus propranolol versus propranolol alone for the primary prophylaxis of esophageal variceal bleeding. Clin Mol Hepatol. 2014;20(3):283-90.
77. Bhardwaj A, Kedarisetty CK, Vashishtha C, et al. Carvedilol delays the progression of small oesophageal varices in patients with cirrhosis: a randomised placebo-controlled trial. Gut. 2016.

78. Kim JH, Sinn DH, Kim K, Kang W, Gwak GY, Paik YH, et al. Primary prophylaxis for variceal bleeding and the improved survival of patients with newly diagnosed hepatocellular carcinoma. Dig Dis Sci. 2016;61(11):3354-62.

79. Reiberger T, Mandorfer M. Beta adrenergic blockade and decompensated cirrhosis. J Hepatol. 2017;66(4):849-59 A comprehensive review on potential deleterious effects of NSBB therapy in advanced/decompensated cirrhosis.

80. Garcia-Tsao G, Sanyal AJ, Grace ND, Carey W. Prevention and management of gastroesophageal varices and variceal hemorrhage in cirrhosis, Hepatology (Baltimore MD 2007;46(3):922-938.

81. Bosch J. Carvedilol: the beta-blocker of choice for portal hypertension? Gut. 2013;62:1529-30.

82. Sinagra E, Perricone G, D'Amico M, Tine F, D'Amico G. Systematic review with meta-analysis: the haemodynamic effects of carvedilol compared with propranolol for portal hypertension in cirrhosis. Aliment Pharmacol Ther. 2014;39(6):557-68.

83. Mandorfer M, Peck-Radosavljevic M, Reiberger T. Prevention of progression from small to large varices: are we there yet? An updated meta-anaylsis. 2016.

84. D'Amico G, Pagliaro L, Bosch J. Pharmacological treatment of portal hypertension: an evidence-based approach. Semin Liver Dis. 1999;19(4):475-505.

85. Villanueva C, Aracil C, Colomo A, Hernández-Gea V, LópezBalaguer JM, Alvarez-Urturi C, et al. Acute hemodynamic response to beta-blockers and prediction of long-term outcome in primary prophylaxis of variceal bleeding. Gastroenterology. 2009;137(1):119-28.

86. La Mura V, Abraldes JG, Raffa S, et al. Prognostic value of acute hemodynamic response to i.v. propranolol in patients with cirrhosis and portal hypertension. J Hepatol. 2009;51(2):279-87.

87. Hernandez-Gea V, Aracil C, Colomo A, et al. Development of ascites in compensated cirrhosis with severe portal hypertension treated with beta-blockers. Am J Gastroenterol. 2012;107(3):41827.

88. Groszmann RJ, Bosch J, Grace ND, Conn HO, Garcia-Tsao G, Navasa M, et al. Hemodynamic events in a prospective randomized trial of propranolol versus placebo in the prevention of a first variceal hemorrhage. Gastroenterology. 1990;99(5):1401-7.

89.• Villanueva C, Albillos A, Genescà J, et al. Preventing the decompensation of cirrhosis with $\beta$-blockers in patients with clinically significant portal hypertension. A multicenter double-blind placebo-controlled randomized clinical trial. Hepatology. 2016;64(6): $1121 \mathrm{~A}-2 \mathrm{~A}$ This study provides evidence that HVPG-guided NSBB therapy is prevents hepatic decompensation in compensated patients with CSPH, but without VNT.

90. D'Amico G, Pasta L, Morabito A, D'Amico M, Caltagirone M, Malizia G, et al. Competing risks and prognostic stages of cirrhosis: a 25-year inception cohort study of 494 patients. Aliment Pharmacol Ther. 2014;39(10):1180-93.

91. Serste T, Melot C, Francoz C, et al. Deleterious effects of betablockers on survival in patients with cirrhosis and refractory ascites. Hepatology. 2010;52(3):1017-22.

92. Mandorfer M, Bota S, Schwabl P, et al. Nonselective beta blockers increase risk for hepatorenal syndrome and death in patients with cirrhosis and spontaneous bacterial peritonitis. Gastroenterology. 2014;146(7):1680-1690 e1681.

93. Serste T, Njimi H, Degre D, et al. The use of beta-blockers is associated with the occurrence of acute kidney injury in severe alcoholic hepatitis. Liver Int. 2015;35(8):1974-82. 